\title{
Automatic Calibration of Ultra Wide Band Tracking Systems Using A Mobile Robot: A Person Localization Case-study
}

\author{
Alessio Canepa, Zeynab Talebpour and Alcherio Martinoli
}

\begin{abstract}
Ultra Wide Band (UWB) is an emerging technology in the field of indoor localization, mainly due to its high performances in indoor scenarios and relatively easy deployment. However, in complex indoor environments, its positioning accuracy may drastically decrease due to biases introduced when emitters and receivers operate in Non Line-of-Sight (NLOS) conditions. This undesired phenomenon can be attenuated by creating, a priori, a map of the measurement error in the environment, that can be exploited at a later stage by a localization algorithm. In this paper, the error map is the result of a calibration process, which consists of collecting several measurements of the localization system at different locations in the environment. This work proposes the leveraging of mobile robots in order to automatize the calibration process with the ultimate purpose of improving UWB-based people localization in a realistic indoor environment. The whole process exploits existing algorithms in the field of robot localization conveniently adapted in order to address our use case and technology. Experiments in real environments of incrementally increasing complexity show how the average localization accuracy can be improved up to $50 \%$ by adopting this method.
\end{abstract}

Index Terms-Localization, Calibration and Identification.

\section{INTRODUCTION}

Nowadays, the ability to track objects and people is of essential importance for an ever increasing number of applications. So far, the revolution brought by Global Positioning System (GPS) has indeed been able to satisfy this need in open environments, with an average accuracy of $5 \mathrm{~m}$ using consumer grade devices [1]. Over the recent years, the relocations of many activities from outdoor to indoor environments, together with the advent of new technologies, has opened the doors to the new field of indoor localization, where Global Navigation Satellite Systems (GNSS) in general are not suitable.

Most indoor localization systems are based on radio technologies such as WLAN, Bluetooth, RFID and UWB. One key characteristic of such technologies is that unlike camera-based systems, they do not require Line-of-Sight (LOS) between the tracking sensors and the tracked objects. Given the focus of this work, we review the radio-based indoor tracking literature distinguishing in particular between the non-fingerprinting and fingerprinting-based approaches. A survey on mathematical methods for indoor localization can be found in [2].

Non fingeprinting-based solutions such as [3], [4] are capable of computing the position of the target without any kind of a priori information. Most common systems of this kind measure the distance between the target and one or multiple base stations through RSS (Received Signal Strength) readings of a WLAN network. As an example, in [4] a WLAN RSS-based localization algorithm is presented that achieves roughly $70 \mathrm{~cm}$ of accuracy in a dense multi-room environment. However, these solutions are affected by unpredictable signal attenuation which significantly compromise their accuracy in real scenarios.
Fingerprinting-based solutions adopted in many studies such as [5]-[7], significantly improve the tracking accuracy by using scene analysis techniques. In the first phase which is offline, several measurements of the localization system called fingerprints are collected at various reference locations in order to create a "radio map" of the environment. In the second phase which is online, the previously collected data is exploited by a localization algorithm.

A fingerprinting-based probabilistic approach for UWB localization, proposed by Prorok et al. [8] is a key reference for this work and will be analyzed in the next section. We note that in this paper, the term fingerprinting differs from what is commonly used in the literature, while following the same principles. In this work, during the off-line phase of fingerprinting, error models for individual grid cells are extracted. These models are then used in the online phase for assigning weights to corresponding position candidates. In other words, there exists a mapping between any given position and an error model which will be used for weighting the importance of that position candidate.

Between all the radio-based tracking systems, our work focuses on UWB. As shown by the comparisons carried out by Rejane et al. [9] and Disha et al. [10] on indoor positioning technologies, UWB is particularly interesting due to several reasons, including high accuracy, material penetrability, large coverage and scalability. A recent survey on the topic can be found in [11].

The basic architecture of an UWB localization system is made up of two components:

- Tags: movable sensors attached to the entity to be tracked; usually battery powered.

- Anchors: sensors placed at known locations; used as a reference to compute the position of the tags.

Radio signals, in the form of pulses, are exchanged between the tracked tag and multiple anchors. On the basis of this principle, various measurement models exist [12]. Among them, we cite the Time Difference of Arrival (TDOA) and the Time of Arrival (TOA). The former calculates the time difference of arrival of the signal to the different anchors, the latter calculates each tag-anchor distance on the basis of the signal travel time. In both cases, the tag's position is estimated by fusing all the measurements with a localization algorithm [12].

In outdoor environments, modern UWB localization systems manage to achieve centimeter-scale accuracy in areas as large as thousands of square meters [13]. On the contrary, in indoor environments, these systems are affected by signal attenuations and multi-path phenomena, which are particularly strong in Non-Line-Of-Sight (NLOS) conditions, that means the presence of obstacles on the tag-anchor path. For this reason, most of the literature in this field tends to avoid very complex scenarios 
or the number of anchors is increased to eliminate or minimize the occurrence of NLOS. However, even with these precautions, the final accuracy of these systems is hardly better than $0.2 \mathrm{~m}$. In the following paragraphs we will briefly mention some of the relevant state of the art in this topic, which give an idea of the level of accuracy achievable in different setups.

Segura et al. [14] developed a self-positioning system based on UWB TDOA measurements, composed of an UWB receiver board mounted on a robot, and three external UWB emitter beacons at fixed locations. The positioning accuracy is tested (statically) at five different locations in a relatively simple experimental space with artificially added obstacles. They measured errors in the order of $0.20 \mathrm{~m}$ in the regions with NLOS.

A more real and complex environment has been considered in the work of Stelios et al. [15]. They tested the performance of a commercial UWB positioning system in an indoor environment where at each location only two anchors out of four had LOS conditions with the tag. The accuracy of the system, which in full LOS conditions is roughly $0.15 \mathrm{~m}$, dropped to $1.26 \mathrm{~m}$ in their scenario.

In a recent study [16], Eryildirim et al. propose a localization algorithm based on an Extended Target Gaussian Mixture Bernoulli filter that has been tested by fusing the data of multiple UWB sensors. Their algorithm requires multiple sensors to be placed on the target and is capable of handling the occlusions for one or more of the UWB tags very well. Occlusions can easily happen for a single tag placed on a human's body and the use of multiple tags alleviates this problem to a large extent. Their tests have been carried out in an indoor open environment $(5 m \times 7 m)$, with a walking person carrying six UWB tags on his body. Results show great improvements over the single UWB readings, up to $15 \mathrm{~cm}$ of accuracy. However, we underline that the use of multiple UWB tags as well as the deployment in an open space play a significant role in improving the tracking accuracy.

Tiemann et al. [17] addressed the problem of NLOS ranging errors rejection from a lower level perspective. Instead of filtering the range measurements, they worked directly on raw UWB channel impulse response (CIR), using a special device that provides that kind of data. They performed NLOS rejection by setting a threshold on the ratio of the first path compared to the power of the cumulated CIR. The positioning accuracy of their system consisting of eight anchors and one tag, is under $10 \mathrm{~cm}$ in the horizontal plane and under $20 \mathrm{~cm}$ in the three-dimensional space for $95 \%$ of the measurements.

A more statistical approach has been adopted by Jiménez et al. [18], who used a Bayesian filter implemented with a particle filter and a measurement model that takes into account bad measurements and outliers. In particular, they model the TOA range measurement error as a multimodal probability density function calibrated with sparse measurements.

A similar approach for UWB-based RTLS is that of Prorok and Martinoli [8], who proposed, for TDOA systems, an approach based on creating a priori the map of the TDOA measurement error, consisting of several position-dependent multimodal PDFs, in the scenario of interest. They used a commercial UWB localization system, which gives the possibility to access the raw TDOA measurements. The need to perform a very fine grained fingerprinting, in order to achieve a more accurate error model and, therefore, localization performances, makes this process extremely time consuming to be carried out manually. For this reason, they used mobile robots equipped with an UWB tag. During this phase, the position of the robots was accurately tracked by an overhead camera system [19]. At a later stage, the same robots were localized using the live UWB's measurements processed by a Monte Carlo Localization algorithm (MCL) that exploits the error map previously created, together with odometry and a low-cost infrared-based inter-robot relative positioning system. The environment they used is a small laboratory with artificially added obstacles in order to create NLOS conditions. Comparing the native localization performances of the commercial UWB system they used with those obtained by the fingerprintingbased approach, they measured up to $30 \%$ of improvement and an average error in the range of $10-13 \mathrm{~cm}$.

\section{A. Contributions}

The robustness and the high performance achieved by [8], make this approach very interesting for the case of a people localization in realistic conditions. However, this new scenario introduces several additional disturbances and makes the use of precise tracking tools such as overhead cameras less suitable for the fingerprinting phase. In our research, we started from the approach introduced in [8] and developed a method that brings the advantages of their fingerprinting-based approach, with appropriate modifications, to the field of UWB indoor people localization in realistic scenarios. Instead of focusing just on the final system's accuracy, like most of the literature in this field does, our method takes into account factors concerning its real feasibility in common scenarios, such as the cost of the hardware used and the time required for setting up the whole localization system.

Here we summarize the key points of our work, and we underline the differences with [8], along with our contributions:

- Our goal is to use a localization algorithm, calibrated through fingerprint measurements, in order to improve the performance of a people localization system using UWB technology.

- The UWB system we use in our experiments is TOAbased, instead of TDOA-based. To be more precise, we perform two-way unsynchronized ranging (UR) in our experiments as opposed to one-way cable synchronized ranging (CSR). This makes our system approximately ten times cheaper than one based on CSR, and much easier to install, since it does not need synchronization cables between the anchors, at the cost of having less accuracy.

- The fingerprinting is performed autonomously by a mobile robot which is capable of self-localizing and navigating in a known environment. This solution avoids the use of overhead cameras which are typically not available in realistic scenarios and would require prior clearance because of privacy issues. Moreover, it gives the possibility to schedule automatic fingerprinting sessions which could be performed, for example, overnight in a real environment. 
- Unlike [8], the fingerprinting data are at the end used in a setting that is partially different from the one where they were collected. During the fingerprinting phase, the tag was mounted on a robot, whereas, the system was tested for person tracking with the person carrying the tag on his head. This factor introduces additional disturbance, since the behavior of the UWB signal at a specific location might not be exactly the same if the measuring tag is placed on a robot or on a person's head.

- We perform the localization of a person without using any additional source of data (e.g., odometry) using only the UWB system measurements. Compared to the case of robot's localization, the absence of odometry represents a significant disadvantage.

- Our tests are performed in realistic multi-room environments instead of a laboratory arena.

\section{MATERIALS}

\section{A. UWB Real Time Localization System}

The UWB RTLS we used in our work is the Eliko's Kio Ranging. This solution which is based on a Decawave chipset, makes use of UR and requires four anchors. This choice has come after a thorough comparison with the Ubisense Series 7000 system which, despite better performance in LOS conditions, had a worse performance in multi-room environments [20]. The same result has been obtained by Jiménez et al. in [18], who compared in a very large industrial environment the Decawave, Ubisense and BeSpoon technologies, finding out that the first solution is by far the best, in particular, in NLOS conditions.

In the Eliko's solution, the tag "pings" alternatively all the anchors and waits for a response, then it calculates the round trip time of the signal and, from this, the tag-anchor distance. This 2-way UR mechanism makes the system less accurate than those based on CSR and TDOA [21]. On the other hand, it has a much lower cost, and does not need any synchronization between the anchors, avoiding the use of synchronization cables, as CSR systems need. Moreover, the Kio Ranging system does not require any calibration procedure, apart from measuring the 3D coordinates of the anchors in the environment. The physical characteristics of these devices make them extremely portable and easy to mount in a variety of environments. Their dimensions are $85 \mathrm{~mm} \times 55 \mathrm{~mm} \times 18 \mathrm{~mm}$ and they weight less than $20 \mathrm{~g}$.

In the system's version we used, the tag outputs the measured tag-anchors distances via a serial interface at a rate of $4 \mathrm{~Hz}$. The user needs its own machine and localization algorithm in order to calculate the tag's position estimate.

Two different methods have been implemented in this work. The first one performs trilateration, and requires the 3D positions of the anchors and the four tag-anchor distances as the input. In other words, it finds the least squares solution of the system of the following equations:

$$
\sqrt{\left(x-x_{u}\right)^{2}+\left(y-y_{u}\right)^{2}+\left(z-z_{u}\right)^{2}}=r_{u}
$$

where $u$ indicates the anchor, $x, y$ and $z$ are the unknown coordinates of the tag, $x_{u}, y_{u}$ and $z_{u}$ are the coordinates of the $u$-th anchor and $r_{u}$ is the measured tag-anchor distance.

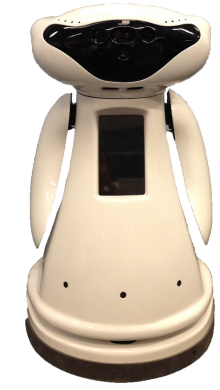

(a)

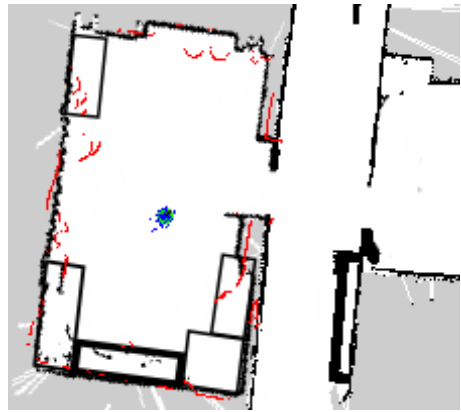

(b)
Fig. 1: a) Robotic platform (MBot) used in our experiments to perform the automatic fingerprinting. b) Graphical view of the robot's position estimation with AMCL. The blue dot is the position estimate, while the red dots represent the measurements of the laser range finders.

The second localization algorithm is the Monte Carlo Localization (MCL) [22]. The main advantage of this method is that it is capable of exploiting the data of the fingerprinting phase and, for this reason, its performances will be compared to those of the trilateration algorithm in order to evaluate the improvements brought by our fingerprinting-based method. The details of this method will be explained in Sec. III.

\section{B. Robot}

The robotic platform used in this work is shown in Fig. 1(a). This robot is called MBot [23] and has been developed within the FP7 European project MOnarCH (Multi-Robot Cognitive Systems Operating in Hospitals ${ }^{1}$ ). It is an omni-directional drive robot with an approximately round footprint of $0.65 \mathrm{~m}$ in diameter and a height of $0.98 \mathrm{~m}$. It is provided with two laser range finders placed on the lower part, between the base and the rest of the robot, on both the front and the back for providing full coverage. Two batteries give the robot an autonomy of approximately five hours, depending on the usage. The UWB tag can be connected via USB to the robot's computer, which runs Ubuntu desktop 12.04 and ROS Hydro. There exist a number of software modules that compose the underlying layers of the robotic platform and provide functionalities such as self-localization and navigation.

The robot's self-localization feature has fundamental importance in our work. It is based on AMCL (Adaptive Monte Carlo Localization) [24], a probabilistic localization algorithm for a robot moving in $2 \mathrm{D}$. AMCL, a variation to the MCL above mentioned, provides an estimate of the position and orientation of the robot by matching the measurements of the laser range finders with a known map of the environment (see Fig. 1(b)) and considering the odometry data. We underline that AMCL has proven to be very robust against unknown obstacles like people (the laser range finders can detect only the legs), bags on the floor and closed/open doors. In the environment of our tests we measured an average self-localization accuracy in the order of $20 \mathrm{~cm}$.

\footnotetext{
${ }^{1}$ http://monarch-fp7.eu/
} 


\section{METHOD}

Our method is an extension of [8] in order to address the problem of people localization in a realistic environment. It consists of three main steps: (i) robotic fingerprinting, (ii) creation of the error map, (iii) localization. In this section, we will explain the mentioned steps in more detail.

\section{A. Robotic fingerprinting}

In this phase, UWB measurements at multiple locations in the scenario of interest need to be collected. Making use of a UR-based UWB system, each measurement $m_{t}$ is in the form of a pair $\left(r_{u}, x_{r}\right)$, where $r_{u}$ is the tag-anchor distance and $x_{r}$ is the position where the measurement has been taken, i.e., the position of the robot performing the fingerprinting at that time.

As mentioned earlier, in [8] the ground-truth of the robot is measured through overhead cameras. The use of overhead cameras to get $x_{r}$ has several limitations: first of all, in a real scenario where multiple rooms have to be scanned, occlusions and limited field of view may call for several cameras, which are expensive, need precise calibration, and have to be all connected to a central computer; secondly, the installation of this kind of system in a public environment may be faced with privacy issues.

Consequently, we use the robot localization data to obtain $x_{r}$. Although this does not provide the same accuracy as overhead cameras, the AMCL-based self-localization system of the robot is a good compromise between accuracy and usability in realworld scenarios.

When performing the fingerprinting the tag was mounted on a structure on the top of the robot, at an overall height of $170 \mathrm{~cm}$. For better performances, different heights should be considered. The consequence of taking all the measurements at a fixed height is that the accuracy of the error map will be maximum for the localization of a person of that height. The fingerprinting path was previously coded in the robot, so that it could follow it automatically. The navigation capabilities of the robot allowed it to adapt the fingerprinting path in case of obstacles. Every $30 \mathrm{~cm}$, the robot stopped and took measurements at different orientations, rotating around its vertical axis.

\section{B. Error map}

The output of the first phase is a large quantity of UR (range) measurements for each anchor and for many different positions and orientations in the environment. The goal of the second phase is to process this data in order to obtain a map of the error. Our error map is divided into squared regions $(1 \mathrm{~m} \times 1 \mathrm{~m})$ and describes the expected UR measurement error (ranging error) in each grid cell and for each anchor.

In more details, this error is described in the form of its probability density function (PDF). The PDF is computed starting from a general error model, in the form of a parametrized error PDF, whose parameters are chosen in order to best fit our measurements.

The error model has been defined starting from [25] as a multimodal PDF. We can formulate it as follows:

$$
p(\Delta r ; \boldsymbol{\theta})=p_{\mathcal{N}}(\Delta r) \cdot P_{L}+p_{\ln \mathcal{N}}\left(\Delta r-\mu_{\mathcal{N}}\right) \cdot\left(1-P_{L}\right)
$$

where $p(\Delta r ; \boldsymbol{\theta})$ indicates the probability density function of measuring an error $\Delta r, p_{\mathcal{N}}(\cdot)$ is a normal distribution of mean

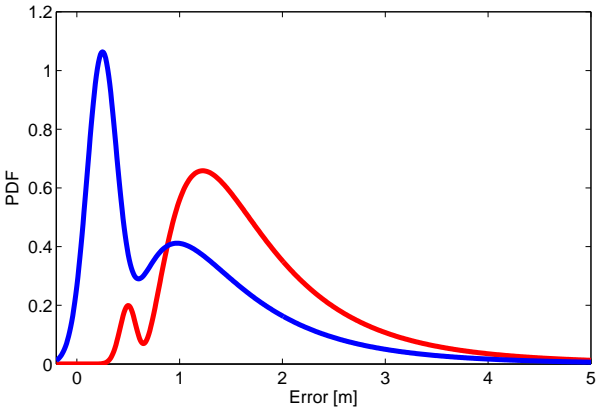

Fig. 2: Examples of error PDF obtained by choosing the parameters of our error model as $\theta_{1}=[0.5,0.08,0.1,0.65,0.04]$ (red) and $\theta_{2}=[0.25,0.15,0.1,0.65,0.4]$ (blue). The red PDF describes the error in an area much more affected by multipath phenomena than the blue one. The red plot is also the PDF of the UR error measured in an experiment described in Sec. V.

$\mu_{\mathcal{N}}$ and variance $\sigma_{\mathcal{N}}, p_{\operatorname{ln\mathcal {N}}}(\cdot)$ is a log-normal distribution of mean $\mu_{\ln \mathcal{N}}$ and variance $\sigma_{\ln \mathcal{N}}$. The value $P_{L}$ is in $[0,1]$ and sets the balance between the normal and the log-normal components. Notice that the log-normal distribution is translated to the right by $\mu_{\mathcal{N}}$. Since $\mu_{\mathcal{N}}$ is the mean of the normal distribution, we can say that it acts as a horizontal bias for the whole PDF. This additional degree of freedom has been added to allow for fitting our measurements to the model more closely.

In simple words, the explanation of this model is the following: the normal part represents the smaller errors measured on the direct path UWB signals; the log-normal part represents the much larger errors that exist due to the multi-path phenomena. Two examples of realizations of our error model are shown in Fig. 2.

$\boldsymbol{\theta}$ is the vector of the parameters of our error model and is defined as:

$$
\boldsymbol{\theta}=\left[\mu_{\mathcal{N}}, \sigma_{\mathcal{N}}, \mu_{\ln \mathcal{N}}, \sigma_{\ln \mathcal{N}}, P_{L}\right]^{T}
$$

Now it should be clear that the error map we want to achieve is in the form of a set of parameters $\boldsymbol{\theta}_{u, v}$, where $u=1 \ldots N_{a}$ specifies one of the $N_{a}$ anchors and $v=1 \ldots N_{r}$ indicates the region index, with $N_{r}$ the total number of regions.

In order to estimate $\boldsymbol{\theta}_{u, v}$, we only consider the fingerprinting measurements taken in the region $R_{v}$ relative to the anchor $A_{u}$. Then, we follow the curve fitting approach presented in [25], adapted by us to suit the UR case, instead of SCR. This approach is a heuristic that estimates the parameters of our error model according to the selected measurements. More details can be found in [8] [20].

\section{Localization}

The third step focuses on localization. This is the only online step of our method. A person walks on a predefined path at constant speed $(\sim 5 \mathrm{~km} / \mathrm{h})$ with a tag on his head. The position of the tag on the top of the head has been chosen after an extensive series of measurements and tests [20], where it was concluded to be the best choice, since it minimizes the probability of the tag being covered by parts of the human body. The tag continuously measures the distance to the four 
anchors at an update rate of $4 \mathrm{~Hz}$. Its measurements are read through its serial interface and stored for later processing. According to [8], an estimate of the tag's position is obtained using MCL [22]. This algorithm uses a particle filter where each particle $\boldsymbol{x}^{[i]}$ represents a position in three dimensions and its weight $w^{[i]}$ is computed considering the UWB measurement and the error map. We configured the height of the particles according to the person's height, which in our case is 170 $\mathrm{cm}$. The number of particles used by the particle filter $M$, is configurable and sets the balance between performance and computational complexity. In our case, 500 particles have been used.

Algorithm 1 shows how MCL works in our application. First, the Initialization is performed by sampling the position of the particles from a bidimensional Gaussian distribution centered on the supposed person's starting point. If the starting point is unknown, MCL can be initialized by spreading the particles uniformly over the environment. After initialization each particle has weight $w_{0}=1 / M$.

Then, the Update function uses the set of UR measures $\mathcal{M}_{t}=\left\{r_{t, 1} \ldots r_{t, N_{a}}\right\}$, taken at time $t$ from all the anchors, to update the weights of the particles according to equations

$$
\begin{gathered}
\Delta r_{t, u}=\left|\boldsymbol{A}_{u}-\boldsymbol{x}_{t}^{[i]}\right|-r_{t, u} \\
w_{t}^{[i]}=\prod_{u=1}^{N_{a}} p\left(\Delta r_{t, u} ; \theta_{u, v}\right)
\end{gathered}
$$

where $\boldsymbol{A}_{u}$ is the known position of the $u$-th anchor and $\theta_{u, v}$ is the vector of the parameters that characterize the error PDF associated to the same $u$-th anchor in the region $R_{v}$, given $x_{t}^{[i]} \in R_{v}$. At the end of the Update function, the position $U_{p}$ of the person is estimated as the weighted average of all the particles.

The next step is the Sample function: a resampling algorithm is used to select which particles to keep and which to discard, according to their weight. In our case, we used the low variance resampling algorithm explained in [26].

Finally, the Move function corresponds to the prediction step of MCL, which aims at changing the position of the particles according to the predicted next position of the tracked object. If available, for instance on a robotic platform, odometry data are used in this step: the particles are moved according to the measured translation of the robot. The lack of odometry data makes people localization much more challenging. Despite several methods such as the Kalman predictor can be used to predict the movement of a person even without external data, in our case we consider the movement of the person as completely random, but limited in speed. For this reason the Move function simply applies a zero-mean Gaussian noise to the 2D position of each particle. The variance $\sigma^{2}$ of this noise has to be chosen according to the sample rate of the algorithm and the supposed maximum speed of the person. In our case we set $\sigma^{2}=0.25$.

In order to test the improvements brought by the use of the fingerprinting data, the localization is computed independently using directly the trilateration algorithm explained in Section II-A (without fingerprinting) and the MCL (calibrated with

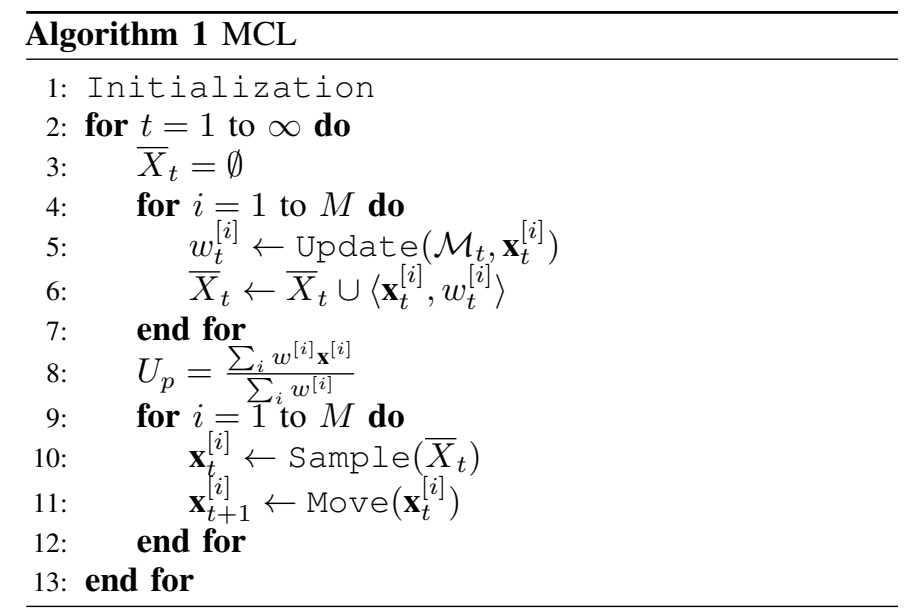

fingerprinting). The same UWB UR measurements collected in our tests were used as input for both algorithms.

\section{SETUP AND EXPERIMENTS}

We considered two different scenarios of incrementally increasing complexity and scope (see Fig. 3). For simplicity we will call them (E1) and (E2). The area of the testing environment for (E1) is approximately $100 \mathrm{~m}^{2}$ while for (E2) it is three times larger. In some locations of (E2) we have up to four walls between the tag and an anchor. This is an extremely challenging condition for a radio-based localization system. Moreover, in (E2) we have on the left-hand side of the corridor (refer to the scheme in Fig. 3) several metallic cabinets, that challenge the UWB system even more. However, all the walls are non-bearing.

We tested also the case of bearing walls in the line between a tag and an anchor and we noticed that this condition makes the performance of the system drastically decrease, probably due to the metallic structural elements they have inside. The choice of such realistic scenarios is given by our goal of considering a real use case with a limited number of anchors in order to keep the cost and complexity of the overall system low, even though we know it has a negative impact on the localization accuracy. The anchors, indicated in the figures with letter A, have been installed at an height of $2.51 \mathrm{~m}$, close to the ceiling. Their position has been measured in our coordinate system very accurately with the help of a laser meter.

For the fingerprinting phase, the robot has been programmed in order to autonomously scan the environment. Roughly 1200 measurements per squared meter have been taken, at 12 orientations around the vertical axis.

During the localization phase a person was walking with a tag on his head along the path indicated by the blue line. In order to record the UR measurements outputted by the tag, the person was walking carrying a bag with a laptop connected via USB to the tag. The groundtruth of the person has been computed by precisely measuring the time taken by the person to walk between the various checkpoints at constant speed. Although, it is theoretically possible to compute the localization estimate online, we did it offline on the basis of the measurements collected. 


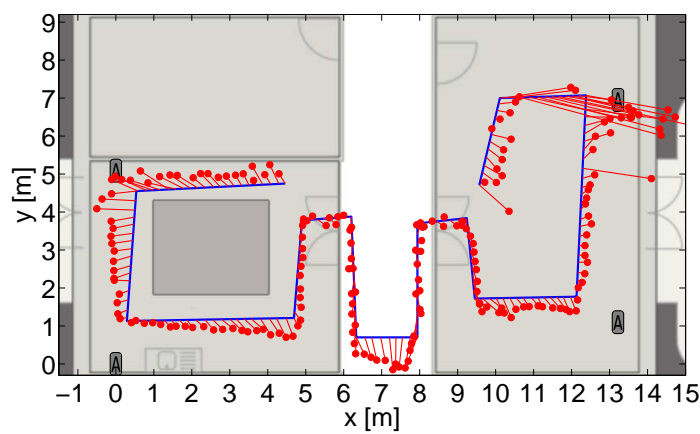

(a) (E1) - Trilateration algorithm

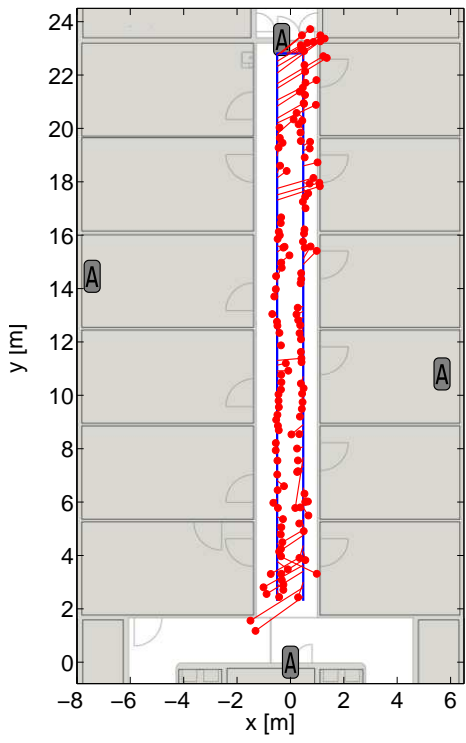

(c) (E2) - Trilateration algorithm

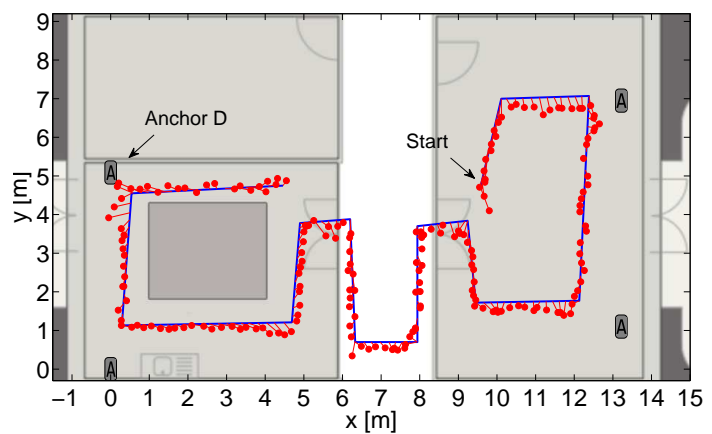

(b) (E1) - MCL calibrated with fingerprints

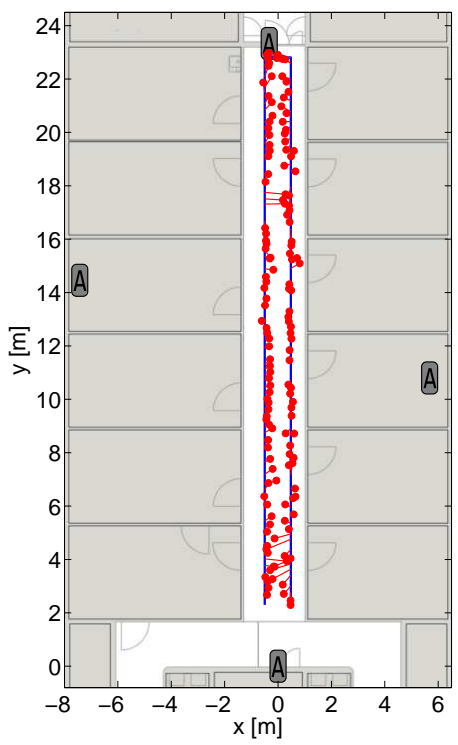

(d) (E2) - MCL calibrated with fingerprints

Fig. 3: Tracking results on first (a-b) and second (c-d) scenarios using the trilateration (a-c) and MCL calibrated with fingerprints (b-d). The blue line is the path of the walking person, while the red lines show the error between the location estimates and their corresponding true positions. Notice how, particularly in the second segment of the path, the accuracy is higher using the fingerprinting-calibrated method.

To have an idea about the accuracy of camera-based tracking solutions compared to the UWB localization methods used in this study, we have made assessments using a marker-based tracking system (SwisTrack) [19] with an overhead camera, and a marker-less solution used in [27] with an omni-directional ceiling mounted-camera. The covering area of the two methods were substantially smaller for a single camera compared to the area covered by the UWB solution. A realistic estimate of the maximum error across the entire arena is in the order of $0.02 \mathrm{~m}$ and $0.2 \mathrm{~m}$ for the aforementioned marker-based and markerless solutions, respectively (refer to Sec. V for comparison). An obvious benefit of using UWB systems is the ability to overcome the field of view limitations of camera-based tracking systems. In this study, we were only allowed to use cameras in the laboratory area due to privacy issues. In general, it can happen in many cases that the entire environment cannot be covered by overhead cameras and hence UWB systems have a significant advantage therein.

\section{RESULTS}

Fig. $3 a$ and $3 b$ compare the results obtained in the first scenario using the trilateration algorithm (a) and MCL calibrated through fingerprinting (b). The red lines show the correspondence between the true position of the person and the estimated one. Let us start our analysis of the results considering the first environment (E1). We can clearly see that, using trilateration, in the second segment of the path the error is particularly high, reaching the maximum value of $3.75 \mathrm{~m}$, while in the rest of the path the average error is $0.36 \mathrm{~m}$.

The red curve of Fig. 2 shows, as resulted by the fingerprinting process, the UR error PDF measured in the second segment of the path in (E1), with respect to anchor D. The interpretation of this PDF is that in the second segment of the path, the distance between the tag and the anchor D is measured with a high positive bias. This behavior is probably due to the presence of multiple walls on the direct path between these two transceivers. This fact also justifies the right bias of the location estimates, as observed in Fig. 3a. 


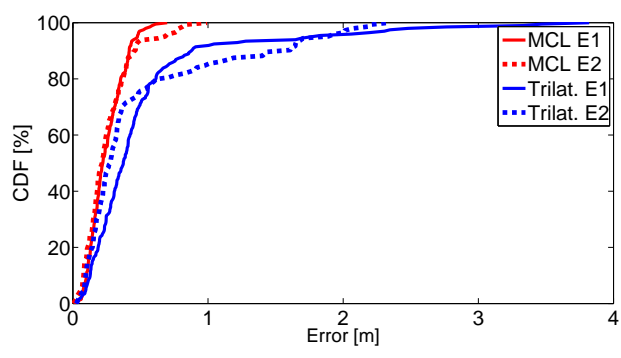

Fig. 4: Cumulative distribution function (CDF) of the error measured in the two scenarios $(\mathrm{E} 1, \mathrm{E} 2)$ using the trilateration algorithm and MCL calibrated via robotic fingerprinting. Notice that with the latter method, the errors above one meter are completely cancelled.

Using the MCL algorithm that exploits the fingerprinting data, we obtained the results shown in Fig. 3b. In particular, we can see that we had a great improvement in the second segment of the path, were the right bias has disappeared and the error has reached the same level as the rest of the path. Considering the whole path, the mean error decreased from $0.51 \mathrm{~m}$ to 0.25 $m$, that is roughly $50 \%$ of improvement. However, given the non-uniform distribution of the error, in Fig. 4 we show its Cumulative Distribution Function (CDF), which gives a better overview of the improvements achieved.

The error CDF shows that our method is less effective against errors lower than $0.30 \mathrm{~m}$, while it works very well against higher errors. This behavior is due to approximations introduced by our general UR error model, the usage of the groundtruth provided by AMCL, the assumption of Gaussian uniform motion model of the human, limited number of measurements in the fingerprinting phase, etc. For many applications, such as people escorting with robots, localizing a person in a circle of $0.50 \mathrm{~m}$ radius is sufficient. Considering this value as our threshold, we measured that our method increased the number of measurements below this threshold from $70 \%$ to $98 \%$ using only a single tag.

The results for the second scenario, are shown in Fig. 3c and $3 \mathrm{~d}$. The anchors are still indicated with the letter "A". We just considered the central corridor and not the rooms, since it is sufficiently complex for our needs.

Comparing the results obtained using the trilateration algorithm and fingerprinting-calibrated MCL, we noticed that, similar to the first scenario, most of the improvements have been brought in the areas which were affected by higher errors: in our case at the top and bottom parts of the map. In the central part of the map, we measured better performances given the lower number of obstacles in the tag-anchor line. The mean error, along the whole path, in the trilateration and MCL cases are respectively $0.48 \mathrm{~m}$ and $0.25 \mathrm{~m}$, confirming an improvement of roughly $50 \%$. The CDF of the error for both the algorithms in this second scenario are shown as the dotted lines, in Fig. 4. Notice that the use of fingerprinting-calibrated MCL made the percentage of localization errors below $0.50 \mathrm{~m}$ increase from $76 \%$ to $94 \%$. Table I summarizes the results discussed so far.

As previously pointed out, MCL is based on a particle filter, which gives the advantages of setting the balance between

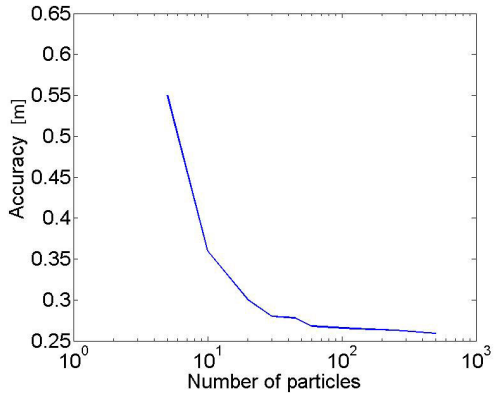

Fig. 5: Analysis of the performance of the MCL localization algorithm as a function of the number of particles used in the particle filter for the second. Notice that increasing the number of particles over one hundred does not significantly improve the performances.

\begin{tabular}{|l|l|l|l|l|}
\cline { 2 - 5 } \multicolumn{1}{c|}{} & \multicolumn{2}{l|}{ Trilateration } & \multicolumn{2}{c|}{ MCL + Fingerpr. } \\
\cline { 2 - 5 } \multicolumn{1}{c|}{} & Average $[\mathrm{m}]$ & $<50 \mathrm{~cm}$ & Average $[\mathrm{m}]$ & $<50 \mathrm{~cm}$ \\
\hline E1 & 0.51 & $70 \%$ & 0.25 & $98 \%$ \\
\hline E2 & 0.48 & $76 \%$ & 0.25 & $94 \%$ \\
\hline
\end{tabular}

TABLE I: Statistics on measurement errors using the two algorithms in both environments.

performance and computational load simply by adjusting the number of particles. In this scenario, we tested the localization algorithm with different number of particles. According to the results shown in Fig. 5, the number of particles significantly affects the performances for values lower than one hundred, while for higher values the improvements tend to stabilize. Therefore, we chose 500 particles for our method, for obtaining a good balance between computational cost and performance.

\section{CONCLUSIONS}

In this work we have seen how in complex indoor environments the accuracy of an UWB RTLS can be significantly improved through a fingerprinting-based calibration method. Moreover, we have seen that this method can be effectively implemented using mobile robots able to autonomously scan the environment.

The major advantages of performing the fingerprinting using mobile robots instead of manually taking measurements are (i) the possibility to take a higher number of measurements and (ii) a more precise association between each measurement and the position where it has been taken and (iii) automatizing a tedious and error-prone job. These factors lead to a more accurate error map, which consecutively brings better performances to the localization procedure. In our tests the use of this method improved the mean error of our system by roughly $50 \%$. In particular, it has shown to be very effective for the rejection of highly inaccurate measurements (errors greater than $0.5 \mathrm{~m}$ ).

Special attention has been given to the feasibility of the proposed method in a real world context: we used a relatively cheap and very easy to install UWB system. The time and work necessary for the fingerprinting phase was acceptable for a real implementation, the environments taken into account were very plausible for this kind of application, and we were able to cover them using just four anchors.

We remind the reader that the localization performances obtained in this work strongly depend on the number of 
measurements taken during the fingerprinting phase and on the accuracy of the robot self-localization system. Improving these two factors would probably lead to even better results. In a real-world scenario, considerable changes in the environment in terms of number of humans, the height at which the tag is placed, placement or removing of large metallic objects, etc., may require recalibration of the localization algorithm. Although this entails additional effort, thanks to the automation of the proposed method, the recalibration process can be performed fairly quickly. The approach presented in this paper can be easily extended to work in three dimensions. Providing the robots with multiple sensors in order to collect data at different heights can be a solution which will not cause the fingerprinting process to take more time. Instead, the computational power needed for MCL will significantly increase, given the larger number of particles that must be considered.

In conclusion, our work has shown how UWB, an emerging technology in the field of RTLS, can be combined with mobile robots and state-of-the-art localization algorithms to provide a complete, cheap and feasible people tracking solution, able to reach an accuracy of approximately $0.25 \mathrm{~m}$ even in realistic indoor scenarios.

\section{ACKNOWLEDGEMENTS}

We thank all the people of the DISAL lab at EPFL and, in particular, José Nuno Pereira, for their support given to this work. We are also grateful to Amanda Prorok, whose work inspired this research and who gave useful hints on how to approach a field she has a lot of experience in. Finally, we are also immensely grateful to Rodrigo Ventura for his assistance and contribution to the navigation components of the software stack of MOnarCH.

\section{REFERENCES}

[1] M. G. Wing, A. Eklund, and L. D. Kellogg, "Consumer-grade global positioning system (gps) accuracy andrreliability," Journal of forestry, vol. 103, no. 4, pp. 169-173, 2005.

[2] F. Seco, A. Jimenez, C. Prieto, J. Roa, and K. Koutsou, "A survey of mathematical methods for indoor localization," Intelligent Signal Processing, pp. 9-14, 2009.

[3] I. Sharp and K. Yu, "Enhanced least-squares positioning algorithm for indoor positioning," IEEE Transactions on Mobile Computing, vol. 12 , no. 8, pp. 1640-1650, 2013.

[4] J. Yang and Y. Chen, "Indoor localization using improved rss-based lateration methods," in IEEE Global Telecommunications Conference, 2009 , pp. $1-6$.

[5] V. Honkavirta, T. Perala, S. Ali-Loytty, and R. Piché, "A comparative survey of wlan location fingerprinting methods," in 6th IEEE Workshop on Positioning, Navigation and Communication, pp. 243-251, 2009.

[6] K. Kaemarungsi and P. Krishnamurthy, "Modeling of indoor positioning systems based on location fingerprinting," in Twenty-third Annual Joint Conference of the IEEE Computer and Communications Societies, vol. 2, pp. 1012-1022, 2004.

[7] P. Bahl and V. N. Padmanabhan, "Radar: An in-building RF-based user location and tracking system," in Nineteenth Annual Joint Conference of the IEEE Computer and Communications Societies. Proceedings, vol. 2, pp. 775-784, 2000.

[8] A. Prorok and A. Martinoli, "Accurate indoor localization with UltraWide Band using spatial models and collaboration," The International Journal of Robotics Research, vol. 33, no. 4, pp. 547-568, 2014.

[9] D. Rejane, V. Thierry, and B. Adrien Van Den, "Comparison of indoor localization systems based on wireless communications," Wireless Engineering and Technology, 2011.
[10] A. Disha, "A comparative analysis on indoor positioning techniques and systems," International Journal of Engineering Research and Applications, vol. 3, pp. 1790-1796, 2013.

[11] A. Alarifi, A. Al-Salman, M. Alsaleh, A. Alnafessah, S. Al-Hadhrami, M. A. Al-Ammar, and H. S. Al-Khalifa, "Ultra-Wide Band indoor positioning technologies: Analysis and recent advances," Sensors, vol. 16, no. 5 , p. $707,2016$.

[12] S. Gezici and H. V. Poor, "Position estimation via Ultra-Wide Band signals," Proceedings of the IEEE, vol. 97, no. 2, pp. 386-403, 2009.

[13] M. Yavari and B. G. Nickerson, "Ultra wide band wireless positioning systems," Dept. Faculty Comput. Sci., Univ. New Brunswick, Fredericton, NB, Canada, Tech. Rep. TR14-230, 2014.

[14] M. Segura, H. Hashemi, C. Sisterna, and V. Mut, "Experimental demonstration of self-localized ultra wide band indoor mobile robot navigation system," in International Conference on Indoor Positioning and Indoor Navigation, pp. 1-9, 2010.

[15] M. A. Stelios, A. D. Nick, M. T. Effie, K. M. Dimitris, and S. C. Thomopoulos, "An indoor localization platform for ambient assisted living using UWB," in Proceedings of the 6th ACM International Conference on Advances in Mobile Computing and Multimedia, pp. 178-182, 2008.

[16] A. Eryildirim and M. B. Guldogan, "A gaussian mixture bernoulli filter for extended target tracking with application to an localization system," Digital Signal Processing, vol. 57, pp. 1-12, 2016.

[17] J. Tiemann, F. Schweikowski, and C. Wietfeld, "Design of an UWB indoor-positioning system for UAV navigation in gnss-denied environments," in Indoor Positioning and Indoor Navigation (IPIN), 2015 International Conference on, pp. 1-7, IEEE, 2015.

[18] A. R. J. Ruiz and F. S. Granja, "Comparing Ubisense, BeSpoon, and DecaWave UWB location systems: Indoor performance analysis," IEEE Transactions on Instrumentation and Measurement, 2017.

[19] T. Lochmatter, P. Roduit, C. Cianci, N. Correll, J. Jacot, and A. Martinoli, "Swistrack-a flexible open source tracking software for multi-agent systems," in 2008 IEEE/RSJ International Conference on Intelligent Robots and Systems, pp. 4004-4010, 2008.

[20] A. Canepa, "Methods for ultra wide band indoor localization using robotic fingerprinting in complex environments," Master's thesis, Politecnico di Torino and École Polytechnique Fédérale de Lausanne, DISAL-MP26, 2015.

[21] C. Zhang, M. Kuhn, B. Merkl, A. E. Fathy, and M. Mahfouz, "Accurate uwb indoor localization system utilizing time difference of arrival approach," in IEEE Radio and Wireless Symposium, 2006.

[22] S. Thrun, W. Burgard, and D. Fox, Probabilistic robotics. MIT press, 2005.

[23] J. Messias, R. Ventura, P. Lima, J. Sequeira, P. Alvito, C. Marques, and P. Carriço, "A robotic platform for edutainment activities in a pediatric hospital," in IEEE International Conference on Autonomous Robot Systems and Competitions, pp. 193-198, IEEE, 2014.

[24] D. Fox, "Kld-sampling: Adaptive particle filters," in Advances in neural information processing systems, pp. 713-720, 2001.

[25] A. Prorok, P. Tomé, and A. Martinoli, "Accommodation of NLOS for ultra-wideband TDOA localization in single and multi-robot systems," in Proceedings of the International Conference on Indoor Positioning and Indoor Navigation, DOI:10.1109/IPIN.2011.6071927, 2011.

[26] S. Thrun, W. Burgard, and D. Fox, Probabilistic Robotics. MIT press, 2005.

[27] Z. Talebpour, D. Viswanathan, R. Ventura, G. Englebienne, and A. Martinoli, "Incorporating perception uncertainty in human-aware navigation: A comparative study," in IEEE Int. Symp. on Robot and Human Interactive Communication, 2016. 